\title{
Presencia de la mujer en las juntas directivas de los clubes de fútbol. Un caso de estudio en la Región de Murcia (España)
}

Presence of Women on the Boards of Football Clubs. A Case Study in the Region of Murcia (Spain)

Marcos Giménez Pérez-Chuecos'

Olga Rodríguez-Ferrán² (0)

1. Alhama C.F. EIPozo.

2. Facultad de Ciencias del deporte. Campus de Escelencia Mare Nostrum.

\begin{abstract}
Resumen
La presencia de la mujer en las juntas directivas se hace difícil debido a que estas organizaciones se crearon para los hombres, dificultando así la presencia y relevancia de la mujer en las mismas. El objetivo del siguiente estudio consiste en examinar la ausencia o presencia de la participación de las mujeres a nivel organizativo (cargos directivos) en la temporada 2019/20 en los clubes de fútbol y fútbol sala de la Región de Murcia. Para el estudio se estilizó como muestra el total de las personas con cargos directivos de los equipos de fútbol y fútbol sala de la Región de Murcia ( $n=1020)$, que con fecha de septiembre del 2019 se encontraban registrados en el Registro Oficial de Clubs Deportivos de la Comunidad Autónoma de la Región de Murcia. Se llevó a cabo una metodología observacional en la que se consideraron como variables: a) modalidad del club (fútbol o fútbol sala); b) género (masculino o femenino) y; c) figura (presidente; vicepresidente 10 ; vicepresidente $2^{\circ}$; vicepresidente $3^{\circ}$; vicepresidente/a $4^{\circ}$; secretario/a; tesorero/a; $7^{\circ}$ vocal; $2^{\circ}$ vocal; $3^{\circ}$ vocal; $4^{\circ}$ vocal; y otros cargos). Los resultados exponen que el porcentaje medio de mujeres en los clubes de fútbol es del $14,28 \%$ y del $21,70 \%$ en los clubes de fútbol sala, además de que, en el 53,36\% de los clubes de la Región de Murcia no hay ninguna mujer en su junta directiva. Se extrae como conclusión que las mujeres aparecen menos que los hombres en los puestos directivos, y que, además, los puestos que ocupan son de menor relevancia dentro de los clubes.
\end{abstract}

Palabras clave: Juntas directivas, participación, fútbol, relevancia, Región de Murcia.

\begin{abstract}
The presence of women on boards of directors is difficult because these organizations were created for men, thus making the presence and relevance of women in them difficult. The objective of the following study is to examine the absence or presence of women's participation at the organizational level (managerial positions) in the 2019/20 season in soccer and futsal clubs in the Region of Murcia. For the study, the total of people with managerial positions of soccer and futsal teams in the Region of Murcia $(n=1020)$ was stylized as a sample, which as of September 2019 were registered in the Official Registry of Clubs Sports of the Autonomous Community of the Region of Murcia. An observational methodology was carried out in which the following variables were considered: a) type of club (soccer or futsal); b) gender (male or female) and; c) figure (president; 1 st vice president; 2nd vice president; 3rd vice president; 4th vice president; secretary; treasurer; 1st member; 2nd member; 3rd member; 4th member; and other positions). The results show that the average percentage of women in soccer clubs is $14.28 \%$ and $21.70 \%$ in futsal clubs, in addition to that, in $53.36 \%$ of the clubs in the Region of Murcia there is no woman on its board of directors. The conclusion is drawn that women appear less than men in managerial positions, and that, in addition, the positions they occupy are of less relevance within the clubs.
\end{abstract}

Keywords: Boards of directors, participation, football, relevance, Region of Murcia.

*Autor de correspondencia: Marcos Giménez Pérez-Chuecos, marcosgimenezpc@gmail.com

Recibido: 14 de diciembre de 2020

Aceptado: 20 de diciembre de 2020

Publicado: 31 de enero de 2021

Como citar (APA): Giménez Pérez-Chuecos, M., y Rodríguez-Ferrán, O. (2021). Presencia de la mujer en las juntas directivas de los clubes de fútbol. Un caso de estudio en la Región de Murcia (España). JUMP, (3), 46-53. https://doi.org/10.17561/jump.n3.6

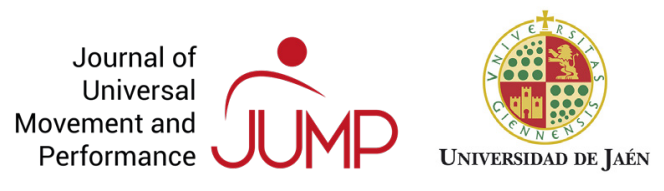




\section{Introducción}

Para poder conocer las relaciones de género que se establecen en las organizaciones se han de conocer los procesos y relaciones que se establecen a diario (Hall, Cullen y Slack, 1989). En general, estas organizaciones se han creado principalmente para el género masculino, es decir, según sus características, facilitándoles así la participación. Por ello, estos organismos deportivos son lugares donde se reproducen los roles de género tradicionales que muestran la gran diferencia de poder que tiene el masculino con respecto al femenino (Claringbould y Knoppers, 2007).

Hay que remontarse hasta el 1994 donde se aprobó la Declaración de Brighton, primer documento internacional sobre el fomento del deporte femenino. A raíz de ahí, numerosas fueron las propuestas que se realizaron en esta línea, destacando principalmente la I Conferencia sobre Mujer y Deporte del Comité Olímpico Internacional, conocido posteriormente como COI (Tusell, 2009). El COI estableció que el porcentaje de mujeres en los cargos directivos tenía que ser de al menos el $10 \%$. Posteriormente, el COl y las Federaciones Internacionales aumentaron dicho porcentaje hasta el 20\% en 2005 (Puig, 2007).

Por su parte, El COE creó en 2004 la Comisión Mujer y Deporte, con el fin de apoyar y contribuir el acceso de las mujeres a los puestos organizativos a nivel deportivo. Según Alfaro et al. (2012), se observó que en el año anterior la participación de las mujeres no llegaba a superar el 10\%. En las juntas directivas la presencia femenina no superaba el $7 \%$ en 2003 , llegando al $7 \%$, porcentaje muy similar al de las Comisiones Delegadas donde en 2003 contaba con un 6,8\% de participación femenina. Este punto de inflexión sirvió para que en el 2006 se pusiera en marcha el Programa Mujer y Deporte, a partir del CSD creando un plan Integral de Deporte, con el fin de incentivar y promover la participación femenina en los cargos directivos. A raíz de esta propuesta se comprobó cómo aumentó la presencia de las mujeres en los puestos organizativos pasando de un 10\% al $12,6 \%$ en 2011 , como en las juntas directivas dicha presencia aumentó de un $7 \%$ a un 10,9\% y se constató el crecimiento de la presencia femenina en las Comisiones Delegadas de un 6,8\% a un $8,11 \%$ en el mismo año (Alfaro et al., 2012). En este sentido, dentro del entorno del fútbol, la FIFA
(2015) está proponiendo y realizando numerosas propuestas para una presencia inmediata de al menos un 20\% delas mujeres en el Comité Ejecutivo de su organismo (confederaciones, clubes, etc.) con un objetivo a largo plazo de aumentar como mínimo este porcentaje hasta el 30\%.

La presencia de las mujeres en los cargos directivos se ha encontrado muy debilitada a nivel deportivo. Como indican Pfister y Radtke (2009) los diversos estudios acerca de este puesto de trabajo en las organizaciones deportivas han demostrado como las mujeres se encuentran menos representadas a niveles de liderazgo y toma de decisiones que los hombres, debido a la poca relevancia que tenían en sus funciones. Según Fernández-Torres y Almansa-Martínez (2011) en 2010 el número de mujeres directivas en el ámbito empresarial, con respecto al fútbol, se encontraba en un $26,9 \%$ del total de trabajadores, habiendo realizado un estudio de más de 72.120 directivas de entre treinta y sesenta años, llegando incluso a destacar que el $70 \%$ de las mujeres se consideran alejadas de la dirección y además un $55 \%$ de ellas consideran que tienen los estudios necesarios como para participar. Estas mismas autoras encontraron que el $95 \%$ de las mujeres entrevistadas señalaron que tenían como superiores a hombres y que solo el $5 \%$ contaba con una mujer en los máximos cargos directivos, y que el 50\% de las mujeres entrevistadas consideraban que el trato que tienen dentro de la organización del club es diferente por el hecho de pertenecer al género femenino.

En este mismo sentido, diferentes estudios señalan que los filtros de acceso que deben pasar las mujeres en los puestos de trabajo, con relación al deporte, son mucho más complicados que los de los hombres (Di Cimbrini et al., 2019; Vega et al., 2019). Conforme a Moragas (2014) y Pfister y Radtke (2009) Ilegaron a la conclusión de que, aunque las mujeres y los hombres tuvieran puestos similares en cuanto a dirección y organización profesional, las mujeres no tenían la misma importancia que ellos, en las recompensas, en la toma de decisión y las juntas directivas. Además, en este estudio los hombres comentaron que no tuvieron ningún impedimento para conseguir el trabajo. Por el contrario, todas las mujeres entrevistadas mostraron su experiencia negativa relacionadas con el impedimento de género marcado por la sociedad, teniendo que compaginar la vida familiar con otro tipo de 
trabajos para poder mantener ese cargo. Estas mujeres concluyeron que debían adaptarse a los modelos tradicionales masculinos impuestos con anterioridad para poder trabajar, debido a que comúnmente se encuentra al hombre como el "eje principal" de todo entramado organizativo, ya fue por un alto nivel socioeconómico, por una vida deportiva plena o por el hecho simple de ser hombre. Como destacaron Vega et al. (2019) las mujeres debían implicarse más que los hombres para considerarlas relevantes en los puestos organizativos debido a que tenían menos recompensa y reconocimiento personal.

Todo esto refleja que el acceso de las mujeres a los cargos directivos no ha sido nada fácil. Las mujeres han tenido que ir superando numerosas etapas hasta llegar al nivel de los hombres, e incluso llegando, se les ha considerado menos relevantes. A raíz de estas etapas, Puig (2007) propone una serie filtros por lo que pasa toda mujer hasta llegar a puestos organizativos en el deporte: personales, de entorno familiar y social y de organización.

En primer lugar, los filtros personales, indica Puig (2007), están relacionados con la falta de confianza que tienen ellas mismas derivando en falta de autoestima y autovaloración; exceso de sentimiento de responsabilidad debido a la poca repercusión de sus trabajos; y, además, la falta de calificación en la esfera pública ya que no están acostumbradas a interactuar con el ente informativo. Asimismo, Adriaanse (2009) otorga un último factor, como es el de la posible falta de formación.

En segundo lugar, para Puig (2007), los filtros asociados al entorno familiar y social están relacionados con la conciliación de lo profesional y lo familiar, debido a que se consideran administradoras de los dos ámbitos. Hay que tener en cuenta las relaciones de género que se producen en los senos familiares donde aún existe una organización patriarcal. Moragas (2014) considera que es posible mejorar este filtro a través de una vida familiar organizada, es decir, disponer de ayuda doméstica y conseguir más tiempo personal.

Por último, el tercer filtro para Puig (2007) está relacionado con la organización. Esta organización se lleva a cabo con el momento actual en el que se encuentra la sociedad. Como se destaca, la mayoría de las asociaciones están contraídas conforme a los aspectos del género masculino por lo que, aunque las mujeres hayan superados los filtros anteriores no se les reconozca su trabajo como tal.

En el fútbol, destaca Pfister (2015), el poder a nivel organizativo o deportivo se encuentra en manos de los hombres. Según Mahmoud (2016), el acceso de las mujeres sigue siendo igual de complicado que en los demás deportes, siendo las exfutbolistas las que más ocupan estos cargos debido a su pasado relacionado con el fútbol. Por un lado, que las mujeres ex profesionales consigan introducirse en los cargos directivos posibilita que haya un conocimiento más amplio acerca del fútbol femenino y de sus participantes, pero también, imposibilita el acceso a aquellas mujeres bien formadas y que no han tenido la oportunidad de jugar de forma profesional.

Según López y Fernández (2018) pocos son los equipos de fútbol que han sido presididos por mujeres. De 1988 a 1992 Alejandra De la Vega presidió al FC Juárez, entre el 1994 y 2011 en el Rayo Vallecano estuvo Teresa Rivero, Ana Urquijo estuvo entre 2006 y 2007 en el Athletic de Bilbao, Rosella Sensi (hija de Franco Sensi) presidió a la Roma de 2008 a 2011 tras el fallecimiento de su padre, en Brasil concretamente en el Flamengo Patricia Amorim estuve al mando durante 2010 a 2012, además Gisela Oeri entre 2006 y 2011 presidió al FC Basilea. En la actualidad muy pocos equipos están presididos por mujeres, solo dos equipos de la liga profesional masculina son dirigidos por mujeres (Eibar y Leganés) y tres en la liga profesional femenina (Málaga femenino, Sporting de Huelva y Atlético de Madrid).

Como se ha comprobado el acceso de las mujeres a los puestos directivos supone un cargo adicional debido a su género, ya que tiene que demostrar en todo momento su capacidad de trabajo y valía. Es por ello, como indica Allison (2016) que cada vez más se está estudiando cómo las mujeres luchan contra la posición de la sociedad y desafían los roles impuestos con el fin de introducirse en el mundo directivo.

Todos estos trabajos exponen la gran dificultad que tienen las mujeres para llegar a los puestos directivos y que se les pueda reconocer su trabajo. En este sentido, es necesario realizar estrategias dirigidas a potenciar la figura de la mujer a través de su trabajo y liderazgo, junto con generar en las mujeres jóvenes el interés por la gestión deportiva. Por ello, el objetivo del presente estudio es analizar la ausencia o presencia de la participación femenina a nivel organizativo (cargos directivos) en los clubs de fútbol de la Región de Murcia. 


\section{Material y método}

La muestra está compuesta por el total de personas con cargos directivos de los equipos de fútbol y fútbol sala de la Región de Murcia $(n=1020)$, que con fecha de septiembre del 2019 se encontraban registrados en el Registro Oficial de Clubes Deportivos de la Comunidad Autónoma de la Región de Murcia.

Los datos que sehan utilizado para la realización de este estudio se han obtenido del registro oficial de la Comunidad Autónoma de la Región de Murcia de la dirección general de deportes. La muestra estaba compuesta por todas las personas con cargos directivos de los equipos de fútbol y fútbol sala de la Región de Murcia.

De cada club deportivo se analizó cada uno de los miembros de la junta directiva inscritos en el registro general, analizando de cada uno de ellos las siguientes variables: a) nombre del club; b) modalidad del club (fútbol o fútbol sala); c) género (masculino o femenino); y d) figura (presidente/a; vicepresidente/a $\quad 1^{0} ; \quad$ vicepresidente/a $2^{\circ}$; vicepresidente/a $\quad 3^{\circ} ; \quad$ vicepresidente/a $\quad 4^{\circ}$; secretario/a; tesorero/a; $1^{\circ}$ vocal; $2^{\circ}$ vocal; $3^{\circ}$ vocal; $4^{\circ}$ vocal; y otros cargos).

Para el registro se siguió el proceso de entrenamiento de observadores en metodología observacional (Losada, y Manolov, 2015). Para calcular la calidad del dato, tras el entrenamiento del observador, se realizó un registro de diez casos, por parte del observador, así como de un observador experto. Se obtuvieron valores de finalidad inter e intra observador mínimo de 1.

Una vez introducidos todos los datos en cuanto a las personas con cargos directivos de los equipos de fútbol y fútbol sala de la Región de Murcia se procedió a un análisis estadístico. Cabe destacar la utilización del programa IBM SPSS Statistics 25.0.

\section{Resultados}

En la tabla 1 se aprecian los datos recogidos con respecto al recuento y porcentaje de hombres, mujeres y su total, en cuanto a presidente, secretario, tesorero y otros cargos, en los clubes de fútbol y fútbol sala de la Región de Murcia.

En la tabla 1, los valores más altos con respecto a fútbol y fútbol sala se encuentran en la categoría de hombre, tanto para presidente, secretario, tesorero y otros cargos, pasando siempre del $70 \%$. El porcentaje de participación femenina se encuentran muy bajo e igualado en todas las categorías, sin superar en ningún caso el $30 \%$.

La información recogida con respecto al porcentaje de mujeres vicepresidentas señala una media de $11,28 \% \pm 31,45 \%$ en fútbol y de $14,47 \% \pm 34,88 \%$ en fútbol sala. De forma semejante se aprecia un porcentaje medio de mujeres vocales de $14,36 \% \pm 32,48$ en fútbol y de $24,75 \% \pm 40,71 \%$ en fútbol sala.

Tabla 1.

Recuento y porcentaje de hombres y mujeres (presidente, secretario, tesorero y otros cargos) en los clubes de fútbol y fútbol sala de la Región de Murcia

\begin{tabular}{|c|c|c|c|c|c|c|c|}
\hline & & \multicolumn{2}{|c|}{ FÚTBOL } & \multicolumn{2}{|c|}{ FÚTBOL SALA } & \multicolumn{2}{|c|}{ TOTAL } \\
\hline & & Recuento & Porcentaje & Recuento & Porcentaje & Recuento & Porcentaje \\
\hline \multirow{3}{*}{ PRESIDENCIA } & HOMBRE & 669 & $91,9 \%$ & 221 & $86,0 \%$ & 890 & $90,4 \%$ \\
\hline & MUJER & 59 & $8,1 \%$ & 36 & $14,0 \%$ & 95 & $9,6 \%$ \\
\hline & TOTAL & 728 & $100,0 \%$ & 257 & $100,0 \%$ & 985 & $100,0 \%$ \\
\hline \multirow{3}{*}{ SECRETARÍA } & HOMBRE & 618 & $85,2 \%$ & 200 & $78,1 \%$ & 818 & $83,4 \%$ \\
\hline & MUJER & 107 & $14,8 \%$ & 56 & $21,9 \%$ & 163 & $16,6 \%$ \\
\hline & TOTAL & 725 & $100,0 \%$ & 256 & $100,0 \%$ & 981 & $100,0 \%$ \\
\hline \multirow{3}{*}{ TESORERÍA } & HOMBRE & 560 & $78,5 \%$ & 187 & $73,9 \%$ & 747 & $77,3 \%$ \\
\hline & MUJER & 153 & $21,5 \%$ & 66 & $26,1 \%$ & 219 & $22,7 \%$ \\
\hline & TOTAL & 713 & $100,0 \%$ & 253 & $100,0 \%$ & 966 & $100,0 \%$ \\
\hline \multirow{3}{*}{ OTROS CARGOS } & HOMBRE & 43 & $81,1 \%$ & 7 & $77,8 \%$ & 50 & $80,6 \%$ \\
\hline & MUJER & 10 & $18,9 \%$ & 2 & $22,2 \%$ & 12 & $19,4 \%$ \\
\hline & TOTAL & 53 & $100,0 \%$ & 9 & $100,0 \%$ & 62 & $100,0 \%$ \\
\hline
\end{tabular}


De manera general en la ilustración 1 se aprecia la media del porcentaje de mujeres que ocupan cargo en las jutas directivas de los clubs de fútbol y /o fútbol sala a del a CARM.

Los datos de la ilustración 1, muestran que el porcentaje medio de mujeres en los puestos directivos es menor que en los clubes de fútbol sala.

En la ilustración 2 se aprecia el número de clubes de fútbol y de fútbol sala en los que hay presencia y/o ausencia de participación de la mujer en las juntas directivas.

Los datos de la ilustración 2, señalan que del total de clubes analizados en el 53,36\% de los clubes no hay ninguna mujer en su junta directiva, en concreto en el $57,22 \%$ de los clubes de fútbol y en el $42,8 \%$ de los clubes de fútbol sala. Por el contrario, en la CARM, existen un total de ocho clubes de fútbol y/o fútbol sala, donde la totalidad de la directiva son mujeres (Agrupación Deportiva Virgen de la Fuensanta, Asociación de Fútbol de Cartagena Féminas, Asociación Deportiva Cotillas
C.F., Asociación Deportiva-Cultural Ciudad del Sol, CEIPS Montepinar, Club Ángeles Celestiales, Club Fútbol Base Jumilla y Racing de la Flota C.F.).

\section{Discusión}

El objeto de este estudio consistió en analizar la presencia de las mujeres en los cargos directivos de los clubes de fútbol y fútbol sala de la Región de Murcia. De forma general, se puede observar la alta cantidad de presencia de al menos una mujer en los cargos directivos de los clubes de fútbol y fútbol sala, destacando que esta presencia es mayor que su ausencia en los clubes de fútbol sala. Como se puede observar la presencia de al menos una mujer es elevada, aunque hay que destacar que la presencia de al menos una mujer no se identifica con la igualdad en cuanto a participación, ya que, en relación con su homólogo masculino este porcentaje baja drásticamente (Vega et al., 2019).

Ilustración 1. Porcentaje de mujeres que ocupan cargo en las juntas directivas.

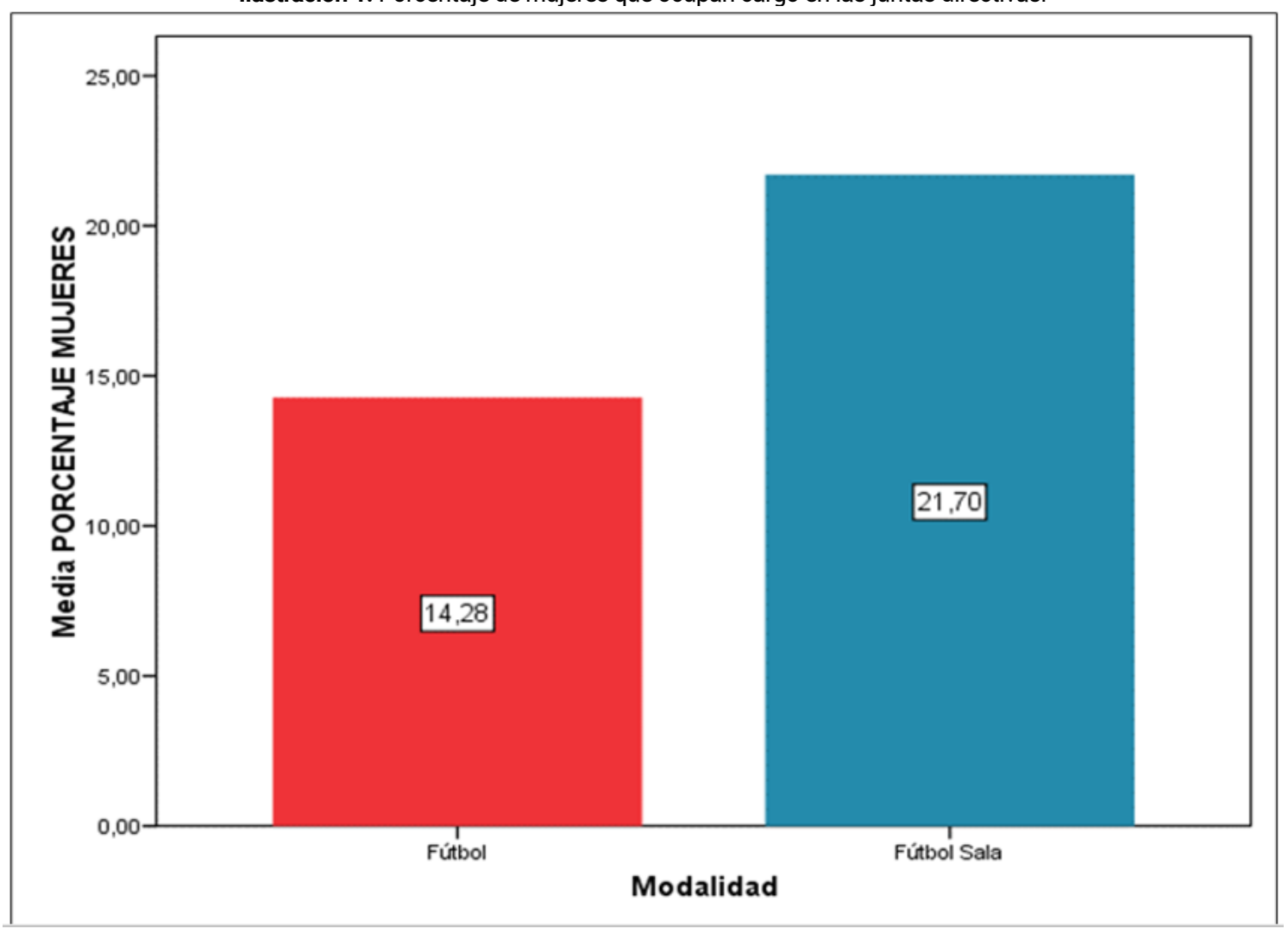


Ilustración 2. Representación del recuento en cuanto a la ausencia o participación de al menos una mujer en los clubes de fútbol y fútbol sala de la Región de Murcia.

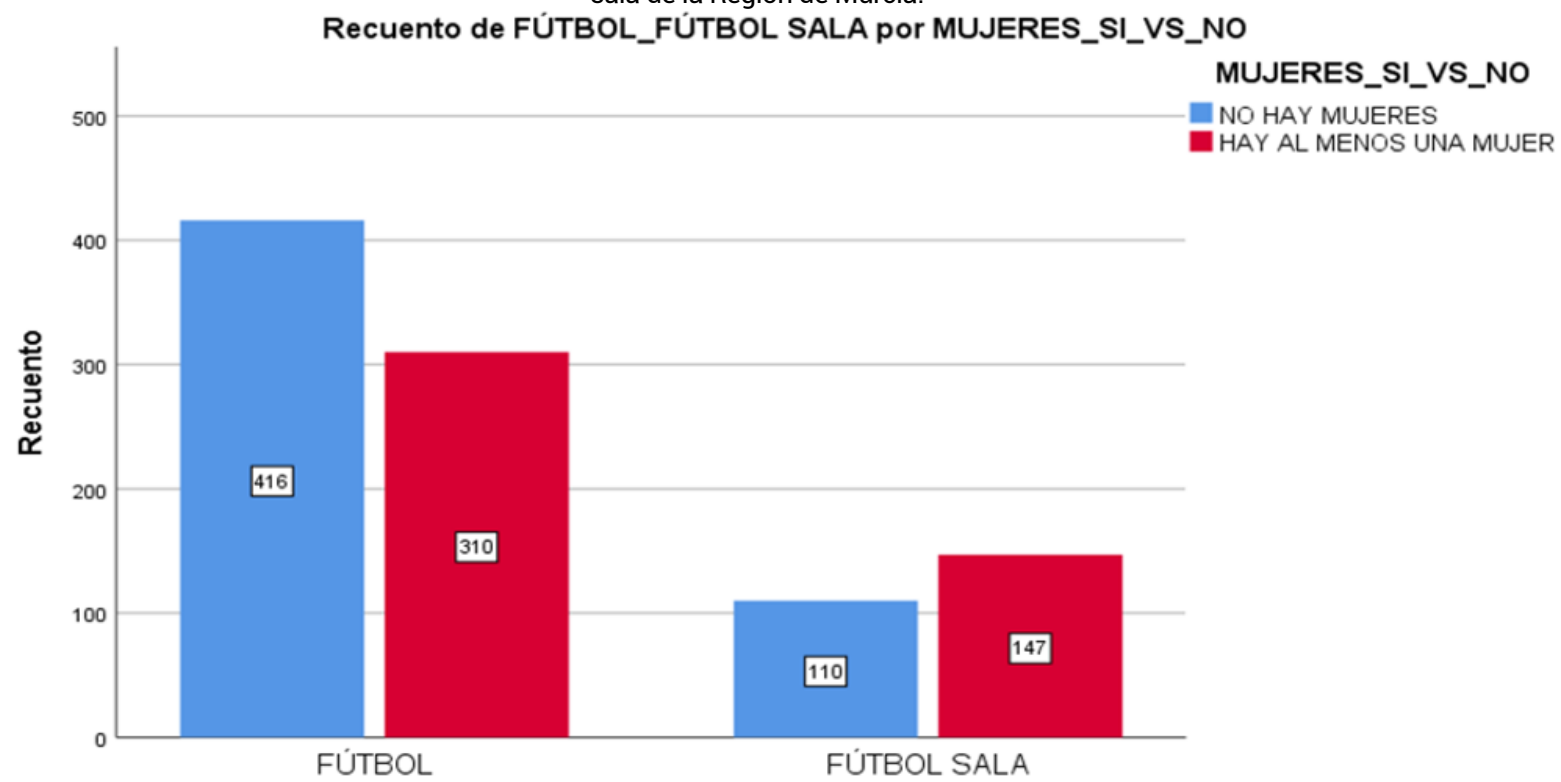

FÚTBOL_FÚTBOL SALA

Con respecto a cargos únicos, es decir, cargos que no se dividen en subcargos (presidente, secretario, tesorero y otros cargos) se puede comprobar que estos no superan el $30 \%$ de participación femenina al igual que se comprobó en el estudio de Chantelat, Bayle y Ferrand (2004). En el cargo de presidente/a el porcentaje femenino es el más bajo de todos los cargos directivos, tanto en fútbol como en fútbol sala, ya que como alega Mahmoud (2016) este cargo suele ser en el que menos mujeres participan en los clubes de fútbol debido a la alta importancia que tiene dentro de la institución. En los puestos de secretario/a, tesorero/a y otros cargos la mujer aumenta su participación en cuanto a número de personas que realizan este trabajo, superando en algunos casos el $20 \%$ y llegando al 26,1\% en fútbol sala. Como se demuestra el acceso de la mujer a este tipo de cargos es mayor que a los cargos donde se toman medidas, ya que, como destacaba Puig (2007) las mujeres suelen tener menos participación en los puestos de representatividad que en los puestos funcionales.

En los cargos que se dividían en varios subcargos, como son el de vicepresidente y vocal, se comprobó la media y la desviación típica a nivel general aportando los siguientes datos: en cuanto a vicepresidentes la media total de fútbol y fútbol sala se encuentra en un $12,12 \%$ de participación femenina y la media de vocal en un 17,08. Aquí se puede comprobar de nuevo como en el estudio realizado por Puig (2007) los cargos representativos de los clubes de fútbol no están presididos por mujeres, dejando a estas otro tipo de cargos de menor importancia. Además, en el estudio realizado por Gambau (2002) se mostró que en Galicia las mujeres representaban un 10\% de participación en cuanto a vicepresidentes y en torno a un $15 \%$ de vocal, una participación muy similar a los clubes de la Región de Murcia. Ambas dispersiones muestran que la participación de la mujer en estos cargos no es muy significativa, por lo que el número de presencia de estas mujeres no suele ser muy elevado.

La información obtenida con respecto al porcentaje total de mujeres en los cargos directivos muestra una media de 18,33\% y una desviación típica de 23,07. Se observa una participación femenina tirando a la baja. Según Mahmoud (2016) en su estudio de los clubes catalanes el $20,7 \%$ de los cargos estaban representados por mujeres. Además, otros estudios realizados en otras juntas directivas de fútbol muestran como el porcentaje femenino de las juntas directivas se encuentra en torno al 22\%, nunca superando el 25\%. (Moragas, 2014). Esto es debido a la falta de oportunidades que tienen las mujeres con respecto al acceso de puestos directivos junto con la falta de reconocimiento de su trabajo, lo que hace que muchas de ellas abandonen (Pfister y Radtke, 2009). 
Diversos estamentos han establecido una serie de condiciones con respecto a la participación de la mujeres en los cargos directivos. La FIFA (2016) estableció un plan de exigencia que plantea que haya al menos un 30\% de mujeres en los cargos directivos en la temporada 2022 y la presencia de al menos una mujer en el 100\% de los cargos directivos en 2026. Según Alfaro, Mayoral y Vázquez (2018) el CSD exige que se aumente la sensibilización e información acerca de las desigualdades entre mujeres y hombres en los cargos directivos, que se fomente el interés de las deportistas en la participación de los puestos directivos, que se lleven a cabo cambios en las estructuras deportivas y que se consiga mejorar las condiciones del entorno sociofamiliar.

En líneas de trabajo futuras se debería realizar numerosas campañas para aplicar las propuestas realizadas por el COI y la FIFA por lo que habría que hacer este tipo de estudios temporada tras temporada con el fin de comprobar la evolución en cuanto a participación femenina en los cargos directivos de los clubes de la Región de Murcia. Además, se debería proponer a nivel nacional que se realizara este estudio con el fin de proporcionar esta información a la RFEF y que pueda trabajar en base a ella con el fin de intentar conseguir una igualdad en este sentido. Por último, otro trabajo interesante que se podría realizar en este ámbito sería recoger la trayectoria y las vivencias de las mujeres durante su trabajo en los puestos directivos, es decir, relevancia dentro de este, acceso al mismo, posibles dificultades, etc.

\section{Conclusión}

Los resultados extraídos estudio $<<$ Presencia de la mujer en las juntas directivas de los clubes de fútbol de la Región de Murcia>> son los siguientes:

- Las mujeres no suelen ocupar los cargos más altos en las juntas directivas.

- El cargo más representado por mujeres en el fútbol de la Región de Murcia es el de tesorero.

- En el fútbol sala las mujeres están más representadas en cuanto a porcentaje se refiere que en el fútbol con respecto a cargos directivos

- La presencia de al menos una mujer en las juntas directivas tiende a igualarse en ambos casos, siendo mayor en fútbol sala.

- La presencia de la mujer se encuentra en 457 clubes entre fútbol y fútbol sala.

\section{Aplicaciones prácticas}

La principal utilidad de este estudio es poder comprobar mediante evidencias científicas la participación y relevancia de la mujer en los puestos organizativos del deporte. Esos datos podrán servir a los estamentos políticos, deportivos y sociales comprobar si realmente las medidas que se están llevando a cabo en los últimos años están teniendo algún efecto relevante a través de la inclusión de la mujer en estos puestos de trabajo, junto con su relevancia en los mismos.

Estos datos deben servir como punto de partida para trabajar sobre ellos, es decir, comenzar a crear temporada tras temporada un proyecto sólido donde la figura de la mujer no sea una mera espectadora. Además, se podrá comparar con otras comunidades tanto el número de puestos de trabajo ocupados por mujeres como la relevancia de los mismos.

\section{Agradecimientos}

Este estudio ha sido realizado gracias a los datos aportados por el registro oficial de la Comunidad Autónoma de la Región de Murcia de la dirección general de deportes.

\section{Referencias}

Adriaanse, J. A. (2009). La mujer y la alta gestión deportiva: de la declaración de Brighton a Sidney 2010. Citius, Altius, Fortius: humanismo, sociedad y deporte: investigaciones y ensayos (CAF), 2(1), 109-128.

Alfaro, É., Mayoral, Á., y Vázquez, B. (2018). Factores que condicionan el acceso de las mujeres a los puestos de responsabilidad en el deporte. Consejo Superior de Deportes.

Alfaro, É., Vázquez, B., Gallardo, J. M., y Ferro, S. (2012). La participación de las mujeres dentro de la Dirección General de Deportes de la Comunidad de Madrid y las federaciones deportivas madrileñas. Kronos, 11(2), 7-16.

Alfaro, É., Vázquez, B., Gallardo, J. M., y Ferro, S. (2012). Mujeres en puestos de responsabilidad dentro de las organizaciones públicas deportivas de la comunidad de Madrid. Ágora para la Educación Física y el Deporte, 15(1), 40-53.

Allison, R. (2016). Business or Cause? Gendered Institutional Logics in Women's Professional Soccer. Journal of Sport and Social Issues, 40(3), 237-262. https://doi. org/10.1177/0193723515615349 
Chantelat, P., Bayle, E., y Ferrand, C. (2004). Les représentations de l'activité des femmes dirigeantes dans les fédérations sportives françaises: effets de contexte et ambivalences. De Boeck Supérieur, 66, 143-159. https://doi.org/10.3917/ sta.066.0143

Claringbould, I., y Knoppers, A. (2007). Finding a 'normal' woman: Selection processes for board membership. Sex Roles, 56(7), 495-507. https://doi.org/10.1007/s11199-007-9188-2

Di Cimbrini, T., Esteban, M. L., Fernandes, E., Güngör, G., y Smith, C (2019). Corporate governance in sport organizations: a gender perspective. Universidad de Zaragoza. https://doi. org/10.26754/uz.978-84-16723-80-5

Fernández-Torres, M.J., y Almansa-Martínez, A. (2011). Mujer y comunicación en el fútbol español. Revista de Comunicación Vivat Academia, 117, 826-842. https://doi.org/10.15178/ va.2011.117E.826-842

FIFA (2015). La FIFA, el fútbol y las mujeres: Razones por las que las reformas deben especificar la inclusión y la inversión. https://resources.fifa.com/mm/document/affederation/ footballgovernance/02/72/23/75/showdoc_es_spanish.pdf

FIFA (2016). Estrategia de fútbol femenino. https://resources. fifa.com/image/upload/women-s-football-strategy. pdf?cloudid=jor8jikrnmjulndmyoip

Gambau, V. (2002). Estudio de la organización de los clubes deportivos en Galicia: un análisis empírico. (Tesis doctoral, Universidade da Coruña). https://ruc.udc.es/dspace/ handle/2183/5595

Hall, M. A., Cullen, D., y Slack, T. (1989). Organizational elites recreating themselves: the gender structure of national sport organizations. Quest, 41(1), 28-45. https://doi.org/10.1080/00 336297.1989 .10483906
Losada, J. L., y Manolov, R. (2015). The process of basic training, applied training, maintaining the performance of an observer. Quality \& Quantity, 49(1), 339-347. https://doi. org/10.1007/s11135-014-9989-7

Mahmoud, M. (2016). El fútbol femenino en los clubes deportivos de la ciudad de Barcelona: un análisis de su gestión. (Tesis doctoral, Universidad de Barcelona). https://www.tesisenred. net/bitstream/handle/10803/405706/MARY\%20MAHMOUD_ TESIS.pdf?sequence $=1$ \&isAllowed $=\mathrm{y}$

Moragas, M. (2014). Les dones que presideixen els clubs esportius a Catalunya. Factors que incideixen en l'accés i en l'exercici del càrrec, i estil de lideratge. (Tesis doctoral, Universidad Ramon Llull). https://www.tesisenred.net/ handle/10803/145029\#page=10

Pfister, G. (2015). Assessing the sociology of sport: On women and football. International Review for the Sociology of Sport Journal, 50(4-5),563-569. https://doi.org/10.1177/1012690214566646

Pfister, G., y Radtke, S. (2009). Sport, women, and leadership: Results of a project on executives in German sports organizations. European Journal of Sport Science, 9(4), 229-243. https://doi. org/10.1080/17461390902818286

Puig, N. (2007). Mujer y deporte. Diputación general de Aragón.

Tusell, J. O. (2009). Deporte y discriminación por sexo: fútbol, remo y salto de esquí. InDeret. Revista para el análisis del derecho, 2(1), 21-35

Vega, P., Pérez-González, B., Fernández-Luna, A., y Burillo, P. (2019). Diversidad de género en las juntas directivas de federaciones deportivas: resultados económicos y operacionales. Apunts. Educación Física y Deportes, 137(3), 115-128. https://doi. org/10.5672/apunts.2014-0983.es.(2019/3).137.09 